\title{
Variability in offset judgments
}

\author{
M. J. PENNER \\ University of Maryland at College Park, College Park, Maryland 20742
}

\begin{abstract}
For brief noise stimuli, Efron has reported data that indicate that perceptual offset occurs approximately $130 \mathrm{msec}$ after stimulus onset. For auditory stimuli, his data were based on the performance of two observers. Using two subjects, Penner repeated Efron's procedure and obtained data that did not replicate his findings. Because the data base is scant and divergent, We extend it by investigating the perceptual offsets of 19 observers. For these observers, the relation between the time of stimulus onset and the perception of offset is quite variable, suggesting the importance of bias or strategy in such tasks.
\end{abstract}

Efron (1970a) devised a technique to measure the time of occurrence of the perception of offset. His observers were presented with a test stimulus (a noise burst of variable duration) and an index stimulus (a 500-msec $2,000-\mathrm{Hz}$ tone) and adjusted these stimuli so that the onset of the index marked the offset of the test stimulus. The interval between the index and the test was taken to be a measure of the time at which the test stimulus was perceived as being off. Rather surprisingly, for brief stimuli, Efron found that the perception of offset occurred at a fixed interval of time after the stimlus onset. On the basis of this result, he hypothesized that perception of a stimulus has minimum duration. Efron presents results for only two subjects whose data are in near agreement with each other.

Reasoning from psychophysical data, Penner (1975) presented an alternative to Efron's hypothesis of a minimum duration. Psychophysical data obtained from forward-masking studies allow one to infer the auditory system's continuing response to a noise burst after the noise has terminated. Forward masking is the masking of a signal by a noise that occurs before it. If the signal is masked, there is a residual effect of the noise: The greater the masking, the greater the residual may be assumed to be. Forward masking occurs for as long as $100-200 \mathrm{msec}$ after a noise has terminated (Duifhuis, 1973; Elliott, 1971; Penner, 1974). More masking occurs if the signal is near the masker in time than if it is far removed from the masker. Forward-masking data are consistent with the view that the effect of a noise decays monotonically during the $100-$ to $200-\mathrm{msec}$ interval following its offset. Data from studies of the detection of silent intervals in noise may also be interpreted as measuring the rate of decay of sensation (Penner, 1977; Plomp, 1964). These data also show that the effect of the noise decays monotonically for $100-200 \mathrm{msec}$ following its physical offset.

This research was supported by a grant from the National Institute of Health. I would like to thank Lynda Nelson and Rhona Bossin for running the subjects and Michael Gottlieb for writing the program.
It seems reasonable to suppose that the perception of the offset of a noise burst is linked to the auditory system's physical responses to that noise. It may be that the perception of offset involves the subject's personal choice, or criterion, as to which level of decay he chooses to associate with offset. If so, perception of offset may occur as soon as the response to the noise begins to decrease, or it may occur when the response has terminated, or perhaps at some point between these extremes. These arguments were the basis for a model proposed by Penner (1975), in which the time of perception of offset is determined by the subject's placement of his criterion. If offset is judged to occur as soon as the noise begins to decay, the model predicts that perception of offset occurs at a fixed interval of time after stimulus onset. Other data are also possible, depending on the criterion. To test this model, Penner repeated the Efron (1970a) procedure using two observers and obtained data that were not identical to his findings. The two subjects displayed distinctly different relations between stimulus-onset times and the perception of offset, indicating the adoption of differing criteria.

Unfortunately, neither Efron nor Penner reported the performance of a sufficient number of subjects to address the issue of whether large intersubject variability is typical. This issue is important because the criterion model implies that the variability will be large when there is no feedback. On the other hand, if the minimum duration is of nearly invariant length as Efron (1970a, 1970b, 1970c) argues, then intersubject variability should be small. In the present research, we will replicate the original Efron paradigm. Our goal in doing this is to determine whether the intersubject variability is large enough to support a criterion-based model.

\section{METHOD}

\section{Subjects}

There were 19 subjects. None of them had ever participated in psychophysical experiments and they had no knowledge of the purpose of this experiment. They were all undergraduates at the University of Maryland and, by running as subjects, they 
were completing their introductory psychology subjectparticipation requirement.

\section{Equipment}

The stimuli consisted of a gated burst of white noise (1381 Random Noise Generator, General Radio) and a gated coherent sinusoidal tone burst of $2,000 \mathrm{~Hz}$. The stimuli were presented dichotically at the same level used by Efron $(1970 \mathrm{a}, 72 \mathrm{~dB}$ SPL at $300 \mathrm{msec}$ ) and were delivered to the subjects over TDH-39 headphones. The burst of white noise, called the test stimulus, was presented to the left ear and the tone burst, called the index stimulus, to the right. The tone was always $500 \mathrm{msec}$. The presentation of stimuli was controlled by a PDP-8E computer. As the duration of the noise increased, its amplitude was decreased by an attenuator (Daven, Type T693) to maintain approximate equality of loudness. Equality of loudness was maintained by decreasing the noise level by $5 \mathrm{~dB}$ per tenfold increase in noise duration for noises less than $100 \mathrm{msec}$ in duration. The loudness was adjusted to follow Efron's procedure. There are questions concerning whether the loudness should be adjusted or whether the noise spectrum level should remain constant. However, since we wish to demonstrate variability in offset judgments rather than to specify the effect of noise level on these judgments, our procedure seems justified.

\section{Psychophysical Procedures}

A modified method of limits was used to obtain the mean point of subjective simultaneity of offset-onset judgments (MSS). The subject was first presented with a stimulus pattern that produced an unequivocal response of temporal gap. For offset judgments, the interval between the offset of the test and the onset of the index stimulus was then reduced by 10 -msec steps until the subject felt that the tone turned on just as the noise went off. The deviation from true simultaneity was recorded and the threshold was then obtained with the index preceding the test stimulus. The mean of these two values was recorded as the threshold. The number of steps in each sequence was intentionally manipulated by starting the series with a variable gap between offset and onset. This prevented the subject from inferring how close he was to his last judgment by counting the number of trials.

The order of presentation of 10 stimulus durations $(10$, $30,50,70,90,110,130,150,200$, and $300 \mathrm{msec}$ ) was randomized in groups of 10 . At the completion of 10 judgments at a single duration, the subjects took a rest period that varied from 1 to $3 \mathrm{~min}$. There were 10 such sittings on a single day so that there was one group of 10 measurements for each of 10 stimulus durations.

The subjects were never informed of the error, if any, between their response and the physical simultaneity of the offset and onset of the test stimuli.

\section{RESULTS}

The data are presented in Figure 1. The ordinate represents the time, $\Delta \mathrm{T}$, between the physical offset of the noise and the physical onset of the tone. For example, if $\Delta T$ was zero, then, on the average, all 19 subjects adjusted the stimuli so that the tone began exactly as the noise terminated. The abscissa represents the noise duration and each data point is the average $\Delta \mathrm{T}$ for all 19 subjects. The best-fitting least-squares line has a slope of -.420 and the coefficient of correlation is .969 . It would be misleading however, to claim that this slope characterized all of the individual subjects' data. To emphasize the extent of the variability, Table 1 displays

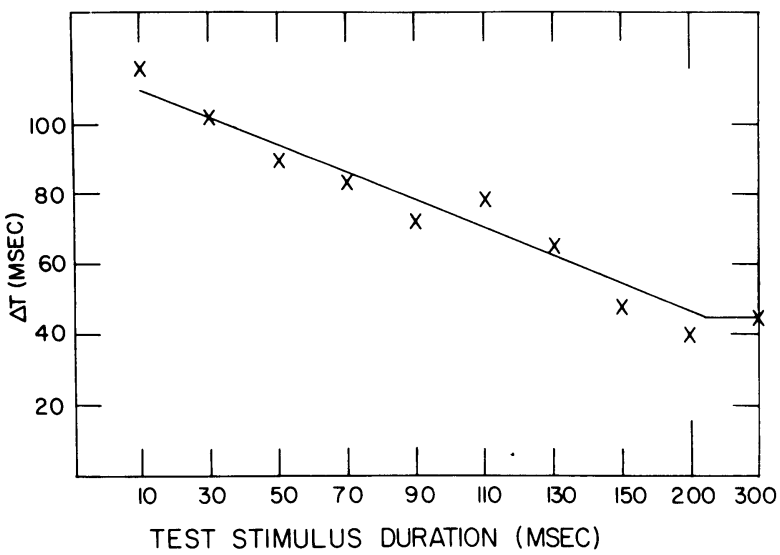

Figure 1. Graph of the deviation from simultaneity, $\Delta T$ (i.e., the time between the offset of the noise and the onset of the tone), as a function of the test stimulus duration. Each data point represents the average $\Delta T$ for all 19 subjects, with 10 estimates of $\Delta T$ for each subject.

the slope of the best-fitting least-squares line for each of the 19 subjects. The slopes range from +.009 to -1.193 . Note that Efron's (1970a, 1970b, 1970c) subjects both obtained a slope of about -1 on these coordinates. His data were well fitted by the function

$$
\begin{aligned}
\Delta \mathrm{T} & =\mathrm{T}-\mathrm{d} & & \mathrm{d} \leqslant \mathrm{T} \\
& =\mathrm{K} & & \mathrm{d}>\mathrm{T},
\end{aligned}
$$

where $\mathrm{d}$ represents the duration of the test stimulus, $\mathrm{K}$ a constant, and $\mathrm{T}$ the minimum duration of the perception. A slope of -1 , then, is necessary in order to interpret these data as evidence favoring a fixed minimum duration of a percept. Note that Efron's results can be found for some subjects (see Table 1).

Table 1

Slope of the Best-Fitting Least-Squares Line

\begin{tabular}{ccc}
\hline $\begin{array}{c}\text { Subject } \\
\text { Number }\end{array}$ & Slope & r \\
\hline 1 & -.383 & .882 \\
2 & +.009 & .032 \\
3 & -.005 & .230 \\
4 & -.573 & .858 \\
5 & -.476 & .753 \\
6 & -.406 & .916 \\
7 & -.274 & .424 \\
8 & -.570 & .760 \\
9 & -.559 & .868 \\
10 & -.023 & .075 \\
11 & -.351 & .672 \\
12 & -.498 & .707 \\
13 & -.357 & .883 \\
14 & -.570 & .942 \\
15 & -.199 & .377 \\
16 & -1.193 & .930 \\
17 & -.324 & .790 \\
18 & -.500 & .716 \\
19 & -.318 & .889 \\
\hline
\end{tabular}

Note-The coefficient of correlation, $r$, is also included. 


\section{DISCUSSION AND CONCLUSION}

If the perception of offset of a noise burst corresponds to some arbitrarily chosen level of the residual stimulation from the preceding noise, then, in the absence of feedback, intersubject variability is expected. The variability should arise simply because there is no reason for all subjects to associate the same residual level with the judgment of offset. The divergent estimates of the slopes of the line relating $\Delta T$ to noise duration (Table 1) indicate that the intersubject variability is, in fact, large. The data do not support the existence of an invariant minimum percept. In terms of modeling the processes involved, then, the present results tend to substantiate the notion that offset judgments are dependent on the subject's personal choice of criterion.

\section{REFERENCES}

Duifhuis, H. Consequences of peripheral frequency analysis for nonsimultaneous masking. Journal of the Acoustical Society of America, 1973, 54, 1471-1488.
Efron, R. Effects of stimulus duration on perceptual onset and offset latencies. Perception \& Psychophysics, 1970, 8, 251-254. (a)

Efron, R. The minimum duration of a perception. Neuropsychologia, 1970, 8, 57-83. (b)

EFron, R. The relationship between the duration of a stimulus and the duration of a perception. Neuropsychologia, 1970, 8, 37-55. (c)

Elliotr, L. L. Backward and forward masking. Audiology, $1971,10,65-76$.

PenNer, M. J. Effect of masker duration and masker level on forward and backward masking. Journal of the Acoustical Society of America, 1974, 56, 179-182.

Penner, M. J. The perception of offset: A problem of decision criteria. Perception \& Psychophysics, 1975, 17, 587-590.

Penner, M. J. Detection of temporal gaps in noise as a measure of the decay of auditory sensation. Journal of the Acoustical Society of America, 1977, 61, 552-556.

PLomp, R. Rate of decay of auditory sensation. Journal of the Acoustical Society of America, 1964, 36, 277-282.

(Received for publication March 14, 1978.) 